\title{
Scapular Upward Rotation During Passive Humeral Abduction in Individuals With Hemiplegia Post-stroke
}

\author{
Jonjin Ratanapinunchai, $\mathrm{PhD}^{1}$, Witaya Mathiyakom, $\mathrm{PhD}^{2}$, Somporn Sungkarat, $\mathrm{PhD}^{1}$
}

\begin{abstract}
${ }^{1}$ Department of Physical Therapy, Faculty of Associated Medical Sciences, Chiang Mai University, Chiang Mai, Thailand; ${ }^{2}$ Department of Physical Therapy, California State University, Northridge, CA, USA
\end{abstract}

\begin{abstract}
Objective To describe scapular upward rotation during passive humeral abduction in individuals with hemiplegia post-stroke compared to normal subjects.

Methods Twenty-five individuals with hemiplegia post-stroke and 25 age- and gender-matched normal subjects voluntarily participated in this study. Scapular upward rotation during resting and passive humeral abduction at $30^{\circ}, 60^{\circ}, 90^{\circ}, 120^{\circ}$, and $150^{\circ}$ were measured using a digital inclinometer.

Results In both groups, scapular upward rotation significantly increased as humeral abduction increased $(\mathrm{p}<0.001)$. Scapular upward rotation was significantly less in the hemiplegic group compared to that in the control at $90^{\circ}(\mathrm{p}=0.002), 120^{\circ}(\mathrm{p}<0.001)$, and $150^{\circ}$ of humeral abduction $(\mathrm{p}<0.001)$. The mean difference in scapular upward rotation between these two groups ranged from $6.3^{\circ}$ to $11.38^{\circ}$.

Conclusion Passive humeral abductions ranging from $90^{\circ}$ to $150^{\circ}$ can significantly alter scapular upward rotation in individuals with hemiplegia post-stroke compared to those of matched normal subjects. The magnitude of reduction of the scapular upward rotation may potentially lead to the development of hemiplegic shoulder pain after prolonged repetitive passive movement. Scapular upward rotation should be incorporated during passive humeral abduction in individuals with hemiplegia post-stroke, especially when the humeral is moved beyond $90^{\circ}$ of humeral abduction. Combined movements of scapular and humeral will help maintain the relative movement between the scapula and humerus. However, further longitudinal study in patients with shoulder pain post-stroke is needed to confirm these findings.
\end{abstract}

Keywords Scapular, Shoulder, Range of motion, Stroke

Received July 3, 2018; Accepted October 16, 2018

Corresponding author: Jonjin Ratanapinunchai

Department of Physical Therapy, Faculty of Associated Medical Sciences, Chiang Mai University, 110 Intawaroroj Rd., Chiang Mai 50200, Thailand. Tel: +66-8-8261-6757, Fax: +66-8-5393-6042, E-mail: jonjin.r@cmu.ac.th

ORCID: Jonjin Ratanapinunchai (http://orcid.org/0000-0002-1166-5011); Witaya Mathiyakom (http://orcid.org/0000-0002-4801-7317); Somporn Sungkarat (http://orcid.org/0000-0003-4283-7469).

(c) This is an open-access article distributed under the terms of the Creative Commons Attribution Non-Commercial License (http://creativecommons.org/ licenses/by-nc/4.0) which permits unrestricted noncommercial use, distribution, and reproduction in any medium, provided the original work is properly cited. Copyright ( 2019 by Korean Academy of Rehabilitation Medicine 


\section{INTRODUCTION}

Hemiplegic shoulder pain (HSP) is one of the most common and disabling complications observed in individuals with hemiplegia post-stroke. The occurrence of HSP varies largely between studies, ranging from $16 \%$ to $84 \%$ [1]. Most patients develop HSP within the first 2 months post stroke [2]. The etiology of HSP includes adhesive capsulitis (50\%), shoulder subluxation (40\%), rotator cuff tears $(22 \%)$, and complex regional pain syndromes (16\%) [2]. Development of HSP has been shown to negatively impact rehabilitation of the affected arm, resulting in impaired activities of daily living $[3,4]$, prolonged hospital stays [5], and delayed functional recovery and quality of life. Reduced passive range of motion of the shoulder particularly in abduction, impaired motor function, and longer duration post stroke have been shown to increase the risk for HSP [6-8]. Therefore, shoulder function and range of motion must be improved and maintained. Evidenced-based treatment and prevention of HSP in individuals with hemiplegia are needed [9].

Passive range of motion exercise (PROME) of the shoulder must be performed correctly to minimize the risk of developing HSP [2]. Many individuals with hemiplegia post-stroke have limited ability to move the affected arm actively. For these individuals, PROME of the affected arm becomes a primary means to maintain shoulder mobility, prevent muscle contracture, and reduce shoulder pain [10-12]. During PROME of the shoulder, the rehabilitation clinician manually supports the affected limb just above the elbow and at the wrist. The rehabilitation clinician then moves the patient's affected limb away from the body through available range [11-13]. Normally, about $60^{\circ}$ of scapular upward rotation is needed to complete $180^{\circ}$ shoulder abduction. In individuals with hemiplegic post-stroke with very weak muscles and abnormal muscle tone, the scapula and its movements are neglected during passive humeral motions. The scapula may not be upward rotated appropriately. Therefore, the glenoid fossa will not elevate sufficiently and the inferior glenohumeral ligament (IGHL) will be stretched as the humerus abducts [14]. This condition may eventually cause the humerus to impinge against the coracoacromial ligament in person without neurological deficit [15]. Therefore, relative motion between the scapula and humerus is needed to maintain the stability of the glenohu- meral joint [13]. However, there is no previous evidence to confirm the movement of scapula during passive shoulder abduction in individuals with hemiplegic poststroke. Understanding scapular motions during shoulder PROME is an essential component of successful treatment strategy to minimize the risk of developing HSP in individuals with hemiplegia post-stroke.

In individuals with normal shoulders, shoulder PROME has been shown to alter the relative motion between the scapula and the humerus or scapula-humeral rhythm $[16,17]$. Significantly less overall scapular upward rotation has been observed in the scapular plane or scaption as compared to the active scaption during passive humeral elevation $[16,17]$. However, differences in scapular upward rotation between active and passive scaption vary between studies [16-18]. Price et al. [18] and Ebaugh et al. [16] have reported no significant differences in scapular upward rotation between active and passive scaption during $0^{\circ}-90^{\circ}$ humeral scaption. However, during $90^{\circ}-160^{\circ}$ humeral scaption, significantly less scapular upward rotation has been noted during passive scaption compared to that during active scaption [16]. In contrast, significantly less scapular upward rotation during $0^{\circ}-80^{\circ}$ passive scaption and more upward rotation during $90^{\circ}-$ $130^{\circ}$ passive scaption compared to those during active humeral scaption are noted [17]. Despite such betweenstudy differences in scapular upward rotation observed between active and passive humeral scaption, results of these studies suggest a significant effect of soft tissue tension generated during arm elevation on the magnitude and timing of scapular upward rotation.

Currently, there is little evidence regarding scapular motions during passive humeral abduction in individuals with hemiplegia post-stroke. Tyson and Chissim [12] have compared two different arm handling methods during passive humeral flexion in individuals with hemiplegia post-stroke. Holding the affected arm near the axilla and maintaining humeral external rotation has resulted in a significantly greater range of shoulder flexion as compared to holding the wrist alone. However, scapular motions during passive humeral flexion are not reported [12]. Hardwick and Lang [13] have reported that scapular upward rotation and humeral external rotation during person-active-assisted shoulder flexion are significantly less in individuals with hemiplegia post-stroke as compared to those of normal controls. Collectively, these re- 
sults further suggest that muscle tension may play a significant role in scapular upward rotation during shoulder PROME.

Limitation of passive humeral abduction is known to a significant risk factor for developing HSP [8]. However, current knowledge of scapular motions during passive shoulder abduction is limited. Thus, the purpose of this study was to describe scapular upward rotation during PROME of shoulder abduction or passive humeral abduction in individuals with hemiplegia post-stroke compared to age- and gender-matched normal controls. It was hypothesized that as the range of passive humeral abduction increased, the magnitude of scapular upward rotation would also increase. However, the increase in upward scapular rotation would be less in individuals with hemiplegia post-stroke as compared to that in normal controls. Scapular upward rotation is known to be the predominant scapular motion during shoulder abduction $[19,20]$. Thus, scapular upward rotation was focused in this study. In contrast, compared to upward rotation, scapular tilt and internal and external rotation are relatively small in range. They are considered as secondary and accessory scapular motions, respectively $[19,20]$.

\section{MATERIALS AND METHODS}

\section{Participants}

Sample size calculation was determined from a pilot study using the magnitude of scapular upward rotation at $30^{\circ}, 60^{\circ}, 90^{\circ}, 120^{\circ}$, and $150^{\circ}$ as the primary outcome. The effect size ranged from 0.94 to 1.53 . Using the lowest effect size of 0.94 , a power of 0.8 , and a significance level of 0.05 , the number of subjects needed for each group was
23. Twenty-five individuals with hemiplegia post-stroke were recruited from physical therapy departments of hospitals in Chiang Mai and Samutprakarn provinces of Thailand. The inclusion criteria for all participants were: (1) no shoulder pain for at least 3 months prior to data collection, and (2) passive shoulder flexion and abduction equal to or greater than $150^{\circ}$. For individuals with hemiplegia post-stroke, they must be able to sit unsupported for at least 5 minutes. The strength of the shoulder and scapular muscles of the affected arm was graded between zero to one on manual muscle testing. Exclusion criteria for all participants were: (1) having any history of injury or surgery to the arm, (2) upper thorax or back that might affect the scapula-humeral rhythm, and (3) marked thoracic kyphosis or scoliosis.

Of 25 individuals with hemiplegia post-stroke, 15 of them had less than normal muscle tone of the shoulder. They were classified as the flaccid group. The remaining ten subjects had minimally increased muscle tone of shoulders, similar to those classified as having 1 to $1+$ Modified Ashworth Score. They were assigned to mildly spastic group. Twenty-five healthy subjects who were age- and gender-matched to subjects in the flaccid and mildly spastic group served as controls. Posture of the thorax is known to affect the scapular motion $[21,22]$. Thus, these subjects were also matched for thoracic kyphosis. Characteristics of participants in this study are summarized in Table 1. This research was approved by the Ethics Committee of the Faculty of Medicine, Chiang Mai University with the Certificate of Approval No. $321 / 2006$.

\section{Instrumentation}

A digital inclinometer (Saunder Digital Inclinometer;

Table 1. Characteristics of participants

\begin{tabular}{lcccc}
\hline & $\begin{array}{c}\text { Flaccid group } \\
(\mathbf{n = 1 5})\end{array}$ & $\begin{array}{c}\text { Mildly spastic group } \\
(\mathbf{n = 1 0})\end{array}$ & $\begin{array}{c}\text { Control group } \\
(\mathbf{n}=\mathbf{2 5})\end{array}$ & p-value \\
\hline Age $(\mathrm{yr})$ & $58.80 \pm 2.12$ & $59.50 \pm 4.31$ & $59.16 \pm 2.09$ & 0.969 \\
\hline Height $(\mathrm{cm})$ & $159.33 \pm 1.30$ & $164.30 \pm 2.09$ & $158.58 \pm 1.84$ & 0.053 \\
Weight $(\mathrm{kg})$ & $61.80 \pm 2.91$ & $61.20 \pm 3.46$ & $50.16 \pm 1.86$ & 0.420 \\
\hline Kyphotic Index & $7.16 \pm 0.75$ & $7.25 \pm 0.71$ & $6.99 \pm 0.42$ & 0.983 \\
Duration post-stroke (day) & $53.40 \pm 11.91$ & $128.80 \pm 53.32$ & - & $0.001^{\text {a) }}$ \\
\hline
\end{tabular}

Values are presented as mean \pm standard deviation.

p $>0.05$ one-way ANOVA.

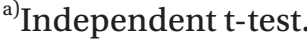


The Saunders Group Inc., Chaska, MN, USA) with a bubble level affixed to it was used to measure scapular upward rotation during passive humeral abduction. This instrument was selected due to its reliability to measure upward rotation position $[23,24]$ and its validity for measuring scapular upward rotation against a sophisticated electromagnetic tracking system [23].

\section{Procedure}

To measure scapular upward rotation, a minimum of two assessors participated in each data collection session. The first author (JR) measured the scapular upward rotation positions in all subjects while the other assessor assisted in passive humeral abduction. During data collection, each participant sat on a modified chair with a sternal support to stabilize the body to maintain participant's posture (Fig. 1A). Two round one-centimeter markers were placed on the medial end of the spine of the scapula and at the posterolateral end of the acromion process. These two markers formed a scapular spine line (Fig. 1C). The angle between the scapular spine line and a horizontal reference line was used to identify scapular upward rotation. In resting position, scapular upward rotation was measured when the tested arm was at the side of the body. After resting trials, a few trials of passive humeral abduction were performed to ensure that sub-
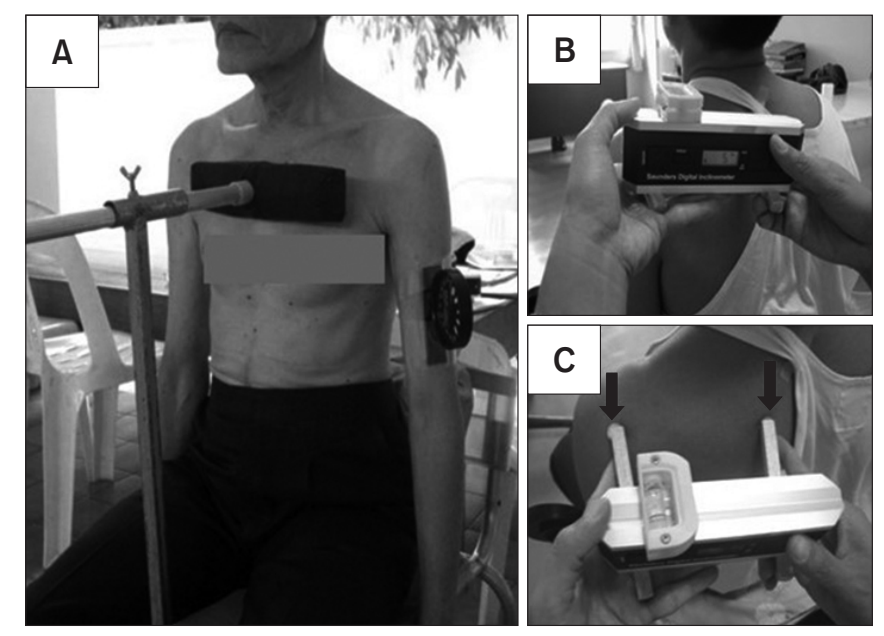

Fig. 1. (A) The setting posture of participants with a sternal stabilizer and a gravitational goniometer to measure glenohumeral abduction. (B, C) Position of a modified digital inclinometer during measuring of scapular upward rotation. (C) Two markers (black arrows) formed a scapular spine line. jects relaxed their tested arm during passive movement prior to measurement. The tested arm was then passively moved to $30^{\circ}$ of humeral abduction using a gravitational goniometer (Fig. 1A). The scapula's markers were moved to the new position while the participant' arm was held steadily by a second assessor. Again, the scapular upward rotation position was recorded using a modified digital inclinometer [23] (Fig. 1B, 1C). The same procedure was then repeated at $60^{\circ}, 90^{\circ}, 120^{\circ}$, and $150^{\circ}$ of humeral abduction. Two trials of data collection were performed at each angle with a 5-minute rest between trials. Data of these two trials were averaged and used for statistical analysis.

\section{Statistical analysis}

Intra-rater reliability and standard error of measurement (SEM) of scapular upward rotation were estimated using data obtained from 25 normal subjects. Intraclass correlation (ICC $[3,2]$ ) was used to identify intra-rater reliability of scapular upward rotation at each passive humeral abduction position. ICC and pooled standard deviations were then used to estimate SEM [25].

Initially, a mixed analysis of variance (ANOVA) was used to identify differences in scapular upward rotation between flaccid and mildly spastic groups. Results showed neither significant ( $p>0.05$ ) main group effects nor group by humeral position interactions. Therefore, data of these two groups were combined and compared to those of the normal control group. As a result, a mixed ANOVA ( 2 groups $\times 6$ humeral positions) was performed for final data analysis. The significance level was set at 0.05. A Bonferroni adjustment was used for post-hoc analysis. All statistical analyses were performed using SPSS version 22.0 (IBM Corp., Armonk, NY, USA).

\section{RESULTS}

There was no significant difference in age, height, weight, or kyphosis index between the stroke group and the control group (Table 1).

\section{Intra-rater reliability and standard error of} measurement

ICC $[3,2]$ was used to identify intra-rater reliability of scapular upward rotation measured using a digital inclinometer. The ICC $[3,2]$ of scapular upward rotation dur- 
Table 2. Intra-rater reliability and standard error of measurement (SEM) in different positions in normal control subjects $(n=25)$

\begin{tabular}{|ccccc|}
\hline Humeral abduction & & Mean \pm SE & ICC (95\% CI) & SEM \\
\hline Resting & Trial 1 & $-1.44 \pm 0.93$ & $0.92(0.81-0.96)$ & 1.33 \\
\hline $30^{\circ}$ & Trial 2 & $-1.84 \pm 0.91$ & & \\
& Trial 1 & $3.08 \pm 0.99$ & $0.91(0.80-0.96)$ & 1.55 \\
\hline \multirow{2}{*}{$60^{\circ}$} & Trial 2 & $3.12 \pm 1.09$ & $0.95(0.89-0.98)$ & 1.36 \\
\hline \multirow{2}{*}{$90^{\circ}$} & Trial 1 & $10.08 \pm 1.27$ & & 1.83 \\
\hline \multirow{2}{*}{$120^{\circ}$} & Trial 2 & $10.48 \pm 1.24$ & $0.95(0.89-0.98)$ & 1.96 \\
\hline \multirow{2}{*}{$150^{\circ}$} & Trial 1 & $24.36 \pm 1.70$ & $0.92(0.83-0.97)$ & 1.85 \\
\hline
\end{tabular}

SE, standard error; ICC, intraclass correlation; CI, confidence interval.

ing passive humeral abduction ranged from 0.91 to 0.95 (Table 2). SEM ranged from $1.33^{\circ}$ to $1.96^{\circ}$ (Table 2). These results indicated good to excellent intra-rater reliability [25] in our measurements of scapular upward rotation using a digital inclinometer. The error of measurement was less than $3^{\circ}$.

\section{Scapular upward rotation during passive humeral abduction}

Scapular upward rotation increased during passive humeral abduction from resting to $150^{\circ}$ in all four groups (Figs. 2, 3). A mixed ANOVA was conducted to compare scapular upward rotation positions between flaccid and mildly spastic groups across six humeral abduction positions. There were no significant main effects of group $\left(F(1,23)=1.573, \mathrm{p}=0.22, \eta_{p}^{2}=0.064\right)$ or interaction effect $\left(F(5,19)=0.742, \mathrm{p}=0.60, \eta_{p}^{2}=0.163\right)$. However, significant main effect of humeral positions was observed $\left(F(5,19)=223.197, \mathrm{p}<0.001, \eta_{p}^{2}=0.983\right)$. Pairwise comparisons indicated significant differences in scapular upward rotation for all humeral abduction positions $(\mathrm{p}<0.001)$. Specifically, scapular upward rotation was significantly greater for the higher humeral abduction position compared to that for the lower one (Fig. 2). Because there were no significant group or interaction effects, data of these two hemiplegic groups were then combined and compared to the control group.

When hemiplegic and control groups were compared, there were significant main effects of group

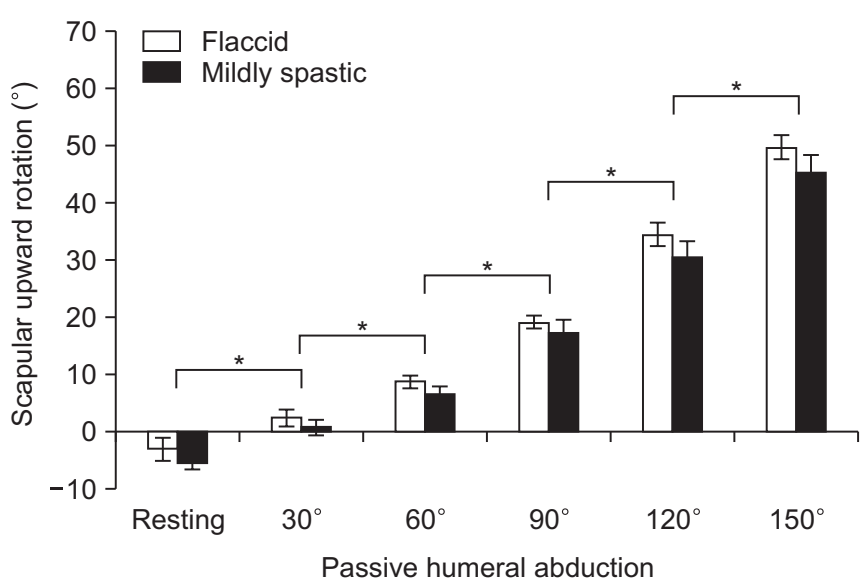

Fig. 2. Comparisons of scapular upward rotation at resting and during passive humeral abduction at $30^{\circ}, 60^{\circ}, 90^{\circ}$, $120^{\circ}$, and $150^{\circ}$ between flaccid group and mildly spastic group. Error bars indicate standard errors of the means. There was no statistically significant difference between the flaccid group and the mildly spastic group. There was a significant difference in scapular upward rotation when compared between humeral positions. ${ }^{*} \mathrm{p}<0.001$.

$\left(F(1,48)=15.331, \mathrm{p}<0.001, \eta_{p}^{2}=0.242\right)$, humeral positions $\left(F(5,44)=676.708, \mathrm{p}<0.001, \eta_{p}^{2}=0.987\right)$, and interaction effect $\left(F(5,44)=4.751, \mathrm{p}=0.001, \eta_{p}^{2}=0.351\right)$. Between-group simple pairwise comparisons demonstrated statistically significant less scapular upward rotation in the hemiplegic group compared to the control at $90^{\circ}(F(1,48)=10.667$, $\left.\mathrm{p}=0.002, \eta_{p}^{2}=0.182\right), 120^{\circ}(F(1,48)=24.73, \mathrm{p}<0.001$, $\left.\eta_{p}^{2}=0.337\right)$, and $150^{\circ}\left(F(1,48)=28.187, \mathrm{p}<0.001, \eta_{p}^{2}=0.370\right)$ 
of passive humeral abduction (Fig. 3, Table 3). The mean difference in scapular upward rotation between these two groups noted during passive humeral abduction from $90^{\circ}$ to $150^{\circ}$ ranged from $6.3^{\circ}$ to $11.38^{\circ}$ (Table 3 ).

\section{DISCUSSION}

This study aimed to identify scapular upward rotation during PROME in the abduction direction in individuals with hemiplegia post-stroke. It was hypothesized that scapular upward rotation during passive humeral abduction would increase as the humeral abduction angle increased. Scapular upward rotation during passive humeral abduction in individuals with hemiplegic post-

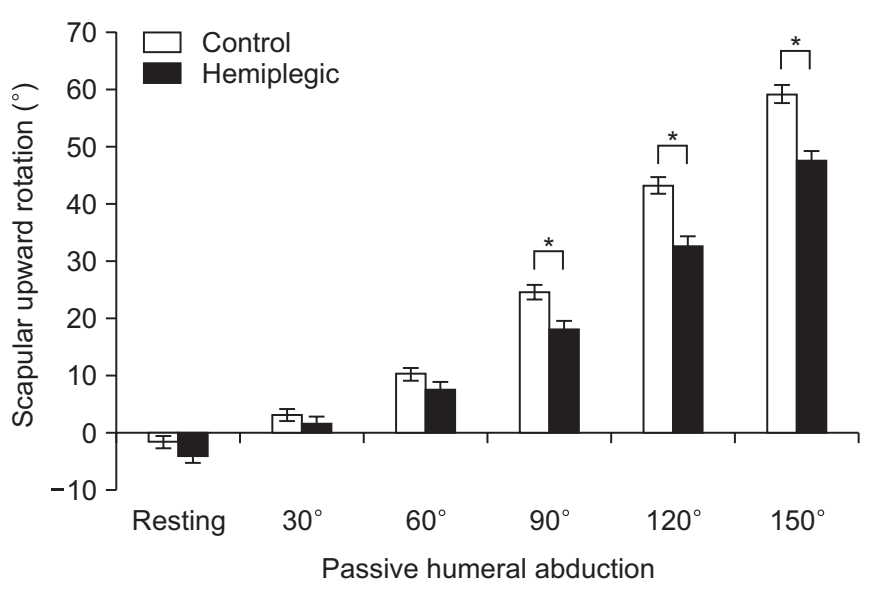

Fig. 3. Comparisons of scapular upward rotation at resting and during passive humeral abduction at $30^{\circ}, 60^{\circ}$, $90^{\circ}, 120^{\circ}$, and $150^{\circ}$ between the control group and the hemiplegic group. Error bars indicated standard errors of the means. Scapular upward rotation was significantly less at $90^{\circ}(\mathrm{p}=0.002), 120^{\circ}(\mathrm{p}<0.001)$, and $150^{\circ}(\mathrm{p}<0.001)$ of passive humeral abduction in the hemiplegic group compared to the control group. ${ }^{*} \mathrm{p}<0.05$. stroke was also hypothesized to be significantly less than that of age- and gender-matched control subjects. Our results confirmed both hypotheses. As the angle of passive humeral abduction increased from 0 to $150^{\circ}$, scapular upward rotation increased in both groups. When the affected arm was at and above $90^{\circ}$ of shoulder abduction, individuals with hemiplegic with flaccid and mildly spastic of the shoulder demonstrated significantly less scapular upward rotation compared to that of match-controls. Additionally, between-group differences in scapular upward rotation were $6^{\circ}-11^{\circ}$, beyond the error of measurement observed in our study. These results signify the need to incorporate scapular upward rotation during passive humeral abduction in individuals with hemiplegic post-stroke.

To our knowledge, this is the first study to demonstrate differences in scapular upward rotation during passive humeral abduction in individuals with hemiplegia poststroke compared to gender- and age-matched controls. These results add to the body of knowledge related to scapular movement during shoulder PROME in individuals with hemiplegia post-stroke. Since a decrease in range of passive shoulder abduction has been shown to be a significant risk factor of developing HSP [8], the ability to improve or maintain shoulder abduction range of motion is of importance for patients with hemiplegic poststroke. Inability to achieve sufficient scapular upward rotation required for arm abduction may lead to abnormal scapulohumeral rhythm, resulting in shoulder injury and pain [2]. Identification of differences in scapular upward rotation during passive humeral abduction will allow rehabilitation clinicians to use a better handling method to incorporate scapular upward rotation during passive humeral abduction in individuals with hemiplegia poststroke.

Table 3. Scapular upward rotation during passive humeral abduction of control and hemiplegic groups

\begin{tabular}{|cccccc}
\hline Humeral abduction & Control group & Hemiplegic group & Difference & 95\% CI of difference & p-value \\
\hline Resting & $-1.64 \pm 1.09$ & $-4.14 \pm 1.09$ & $2.50 \pm 1.55$ & $-0.61,5.61$ & 0.11 \\
$30^{\circ}$ & $3.10 \pm 1.03$ & $1.70 \pm 1.03$ & $1.40 \pm 1.45$ & $-1.52,4.32$ & 0.34 \\
$60^{\circ}$ & $10.28 \pm 1.05$ & $7.80 \pm 1.05$ & $2.48 \pm 1.49$ & $-0.51,5.47$ & 0.10 \\
$90^{\circ}$ & $24.60 \pm 1.36$ & $18.30 \pm 1.36$ & $6.30 \pm 1.93^{*}$ & $2.42,10.18$ \\
$120^{\circ}$ & $43.26 \pm 1.49$ & $32.84 \pm 1.49$ & $10.42 \pm 2.11^{*}$ & $6.18,14.66$ & 0.002 \\
$150^{\circ}$ & $59.26 \pm 1.52$ & $47.88 \pm 1.52$ & $11.38 \pm 2.14^{*}$ & $7.07,15.69$ & $<0.001$ \\
\hline
\end{tabular}

Values are presented as mean \pm standard error. ${ }^{*} \mathrm{p}<0.05$. 
Although this study utilized a simple method to measure scapular upward rotation, this method has been shown to be reliable and valid. In our study, good to excellent intra-rater reliability was found based on our ICC $[3,2]$ values of 0.90 to 0.95 and small error of measurement $\left(<3^{\circ}\right)$. Our data were also consistent with other studies $[23,24]$. Additionally, this method has good to excellent validity against a sophisticated electromagnetic tracking system to measure scapular upward rotation [23]. Given the reliability, small error of measurement, and validity of the method, we believe that between-group differences in scapular upward rotation of $6^{\circ}-11^{\circ}$ observed during $90^{\circ}-150^{\circ}$ of passive humeral abduction are not only statistically significant, but also beyond the error of measurement of this study. They have significant clinical implications.

As expected, scapular upward rotation increased as the humerus was passively abducted in both hemiplegic and normal control groups. This result is consistent with a previous study showing an increase in scapular upward rotation during passive humeral scaption in normal subjects [16-18] and during active-assisted arm flexion performed by individuals with hemiparetic arm [13]. Magnitudes of scapular upward rotation during passive humeral abduction in individuals with hemiplegia poststroke observed in this study are also consistent with those observed during active-assisted humeral flexion in individuals with hemiplegia post-stroke [13]. However, magnitudes of scapular upward rotation during passive humeral abduction in the normal control group are lower than those observed during passive humeral scaption $[16,18]$. These differences in the magnitude of scapular upward rotation can be attributed to differences in the amount of muscle activation used in different types of exercise. It is reasonable to assume that activation of the muscle responsible for scapular upward rotation is greater during person-active-assisted shoulder flexion [13] compared to that during passive shoulder PROME used in our study.

Scapular upward rotation during passive humeral abduction in individuals with hemiplegia post-stroke was significantly less than that in normal subjects when the humerus was at or above $90^{\circ}$ of abduction. Previous studies comparing scapular motions during passive and active scaption in healthy subjects $[16,17]$ have suggested that active tension generated by muscle contraction and passive tension provided by muscle tone with capsuloligamentous structures can contribute to scapular resting position and motion during arm elevation. Impaired muscle tone and activation in individuals with hemiplegia post-stroke are likely to affect scapular motion during passive humeral abduction. Our results of less scapular upward rotation during passive humeral abduction in individuals with flaccid and mildly spastic arm post-stroke compared to normal controls support the role of impaired muscle tone in the reduction of scapular upward rotation during passive arm abduction.

Reduction in scapular upward rotation observed during passive humeral abduction may contribute to HSP in individuals with hemiplegic post-stroke. During active humeral elevation, scapular upward rotation serves to provide stability to the glenohumeral joint and maintain sufficient suprahumeral space [26-28]. Insufficient suprahumeral space observed during passive humeral elevation may lead to shoulder impingement syndrome and rotator cuff tear [26-28]. Our results demonstrated that on average, scapular upward rotation during passive humeral abduction was $6^{\circ}-11^{\circ}$ less in individuals with hemiplegia post-stroke than that in matched controls. Although our study did not compare scapular upward rotation during passive humeral abduction to that during an active abduction, previous studies in healthy subjects indicated significantly less scapular upward rotation during passive humeral scaption compared to that during active scaption $[16,17]$. Taken together, our results suggest that reduction in scapular upward rotation during passive humeral abduction may be even more substantial compared to that of normal controls during an active abduction. As a result, a considerable reduction in scapular upward rotation of $6^{\circ}-11^{\circ}$ observed during passive humeral abduction in individuals with hemiplegic post-stroke signifies a potential for narrowing of the suprahumeral space that may lead to a development of shoulder impingement in the future [29]. However, further studies on patients with hemiplegic shoulder pain are needed to confirm this contention.

Reduction in scapular upward rotation observed during passive humeral abduction may also affect the integrity of soft tissues supporting the glenohumeral joint. During humeral abduction, scapular upward rotation allows the capsule and ligaments around the glenohumeral joint and scapulohumeral muscles to maintain the length and 
tension. It has been shown that the IGHLexperiences the most tension at $90^{\circ}$ of humeral abduction [30]. However, a significant decrease in scapular upward rotation observed during passive humeral abduction at $90^{\circ}$ and above is likely to place the IGHL at risk of being overstretched. Over time, repetitive over-stretching of the IGHL may contribute to an inferior instability and subluxation of the shoulder and pain [30], particularly in the absence of muscular control as observed in individuals with flaccid and mildly spastic arm post stroke.

To minimize the potential for developing of HSP in individuals with hemiplegia post-stroke, scapular upward rotation has been suggested to be incorporated into the shoulder PROME. Currently, methods used for manual PROME in individuals with hemiplegic post-stroke mostly neglect scapular motions [10-13]. Based on results of this study, reduction of the scapular upward rotation during passive humeral abduction may predispose individuals with hemiplegic post-stroke to shoulder impingement and inferior stability, both of which can lead to HSP. Therefore, rehabilitation clinicians and caregivers may need to pay particular attention to scapular movement during passive humeral elevation. In our opinion, it is possible that a rehabilitation clinician can support the patient's affected arm around the elbow and forearm with one hand and use the other hand to grab onto the inferior angle of the scapula. The rehabilitation clinician can then move the scapula passively in an upward rotation direction as the arm is passively elevated. This maneuver must be practiced to ensure comfort and safety of patients.

In conclusion, this study demonstrated a significant decrease in scapular upward rotation during humeral abduction in individuals with hemiplegic post-stroke with very weak muscles and flaccidity or mild spasticity of shoulder muscles compared to healthy controls. The reduction in the scapular upward rotation was more pronounced during $90^{\circ}-150^{\circ}$ of humeral abduction. These results signify the need to incorporate scapular upward rotation during passive humeral abduction in individuals with hemiplegia post-stroke. To confirm benefits of these findings, a longitudinal study is needed to identify the development of HSP.

\section{CONFLICT OF INTEREST}

No potential conflict of interest relevant to this article was reported.

\section{ACKNOWLEDGMENTS}

This study was supported by the Faculty of Associated Medical Sciences, Chiang Mai University, Thailand.

\section{AUTHOR CONTRIBUTION}

Conceptualization: Ratanapinunchai J, Mathiyakom W. Methodology: Ratanapinunchai J, Mathiyakom W. Formal analysis: Ratanapinunchai J, Mathiyakom W, Sungkarat S. Funding acquisition: Ratanapinunchai J. Project administration: Ratanapinunchai J. Writing - original draft: Ratanapinunchai J. Writing - review and editing: Ratanapinunchai J, Mathiyakom W, Sungkarat S. Approval of final manuscript: all authors.

\section{REFERENCES}

1. Snels IA, Dekker JH, van der Lee JH, Lankhorst GJ, Beckerman H, Bouter LM. Treating patients with hemiplegic shoulder pain. Am J Phys Med Rehabil 2002;81:150-60.

2. Lo SF, Chen SY, Lin HC, Jim YF, Meng NH, Kao MJ. Arthrographic and clinical findings in patients with hemiplegic shoulder pain. Arch Phys Med Rehabil 2003;84:1786-91.

3. Roy CW, Sands MR, Hill LD. Shoulder pain in acutely admitted hemiplegics. Clin Rehabil 1994;8:334-40.

4. Wanklyn P, Forster A, Young J. Hemiplegic shoulder pain (HSP): natural history and investigation of associated features. Disabil Rehabil 1996;18:497-501.

5. Roy CW, Sands MR, Hill LD, Harrison A, Marshall S. The effect of shoulder pain on outcome of acute hemiplegia. Clin Rehabil 1995;9:21-7.

6. Blennerhassett JM, Gyngell K, Crean R. Reduced active control and passive range at the shoulder increase risk of shoulder pain during inpatient rehabilitation post-stroke: an observational study. J Physiother 2010;56:195-9.

7. Karaahmet OZ, Eksioglu E, Gurcay E, Karsli PB, Tamkan U, Bal A, et al. Hemiplegic shoulder pain: associ- 
ated factors and rehabilitation outcomes of hemiplegic patients with and without shoulder pain. Top Stroke Rehabil 2014;21:237-45.

8. Lindgren I, Lexell J, Jonsson AC, Brogardh C. Leftsided hemiparesis, pain frequency, and decreased passive shoulder range of abduction are predictors of long-lasting poststroke shoulder pain. PM R 2012;4:561-8.

9. Bender L, McKenna K. Hemiplegic shoulder pain: defining the problem and its management. Disabil Rehabil 2001;23:698-705.

10. Lynch D, Ferraro M, Krol J, Trudell CM, Christos P, Volpe BT. Continuous passive motion improves shoulder joint integrity following stroke. Clin Rehabil 2005;19:594-9.

11. Tseng CN, Chen CC, Wu SC, Lin LC. Effects of a range-of-motion exercise programme. J Adv Nurs 2007;57:181-91.

12. Tyson SF, Chissim C. The immediate effect of handling technique on range of movement in the hemiplegic shoulder. Clin Rehabil 2002;16:137-40.

13. Hardwick DD, Lang CE. Scapular and humeral movement patterns of people with stroke during range-ofmotion exercises. J Neurol Phys Ther 2011;35:18-25.

14. Stefko JM, Tibone JE, Cawley PW, ElAttrache NE, McMahon PJ. Strain of the anterior band of the inferior glenohumeral ligament during capsule failure. J Shoulder Elbow Surg 1997;6:473-9.

15. Sahrmann S. Diagnosis and treatment of movement impairment syndromes. St. Louis: Mosby; 2002.

16. Ebaugh DD, McClure PW, Karduna AR. Three-dimensional scapulothoracic motion during active and passive arm elevation. Clin Biomech (Bristol, Avon) 2005;20:700-9.

17. McQuade KJ, Smidt GL. Dynamic scapulohumeral rhythm: the effects of external resistance during elevation of the arm in the scapular plane. J Orthop Sports Phys Ther 1998;27:125-33.

18. Price CI, Franklin P, Rodgers H, Curless RH, Johnson GR. Active and passive scapulohumeral movement in healthy persons: a comparison. Arch Phys Med Rehabil 2000;81:28-31.

19. Karduna AR, McClure PW, Michener LA. Scapular ki- nematics: effects of altering the Euler angle sequence of rotations. J Biomech 2000;33:1063-8.

20. Ludewig PM, Reynolds JF. The association of scapular kinematics and glenohumeral joint pathologies. J Orthop Sports Phys Ther 2009;39:90-104.

21. Culham E, Peat M. Functional anatomy of the shoulder complex. J Orthop Sports Phys Ther 1993;18:34250.

22. Kebaetse M, McClure P, Pratt NA. Thoracic position effect on shoulder range of motion, strength, and three-dimensional scapular kinematics. Arch Phys Med Rehabil 1999;80:945-50.

23. Johnson MP, McClure PW, Karduna AR. New method to assess scapular upward rotation in subjects with shoulder pathology. J Orthop Sports Phys Ther 2001;31:81-9.

24. Scibek JS, Carcia CR. Assessment of scapulohumeral rhythm for scapular plane shoulder elevation using a modified digital inclinometer. World J Orthop 2012;3:87-94.

25. Portney LG, Watkins MP. Foundations of clinical research: applications to practice. 3rd ed. Upper Saddle River: Pearson; 2009.

26. Flatow EL, Soslowsky LJ, Ticker JB, Pawluk RJ, Hepler M, Ark J, et al. Excursion of the rotator cuff under the acromion: patterns of subacromial contact. Am J Sports Med 1994;22:779-88.

27. Michener LA, McClure PW, Karduna AR. Anatomical and biomechanical mechanisms of subacromial impingement syndrome. Clin Biomech (Bristol, Avon) 2003;18:369-79.

28. Petersson CJ, Redlund-Johnell I. The subacromial space in normal shoulder radiographs. Acta Orthop Scand 1984;55:57-8.

29. Ludewig PM, Cook TM. Alterations in shoulder kinematics and associated muscle activity in people with symptoms of shoulder impingement. Phys Ther 2000;80:276-91.

30. Warner JJ, Deng XH, Warren RF, Torzilli PA. Static capsuloligamentous restraints to superior-inferior translation of the glenohumeral joint. Am J Sports Med 1992;20:675-85. 